\title{
Editorial
}

\section{Has Otolaryngology Subspecialization Gone Too Far in Bangladesh?}

References to the diseases of ear, nose and throat can be traced back to Egyptian, Chinese, Indian and Greek civilization, The Romans, Alexandrines, Arabians and the school of Salerno made important contributions. Hippocrates, the father of medicine who is said to have been born in 460 BC was probably the first to inspect tympanic membrane, 'a dry thin spun-web' and to recognize it as a part of organ of hearing. Laryngology strictly commenced on that day in September 1854 when Manuel Gracia at last achieved lifelong wish to view his own larynx with a mirror. Harmann Khap in 1869 together with Salomon Moos founded 'Archives of Ophthalmology and Otology' in New York. In Vienna where the first university clinic and chair in otology was founded in 1874 , Theodor Billorth, the famous general surgeon stated in 1874, "It is desirable to give this small and yet not unimportant subject a definite place in the curriculum of universities".

'Pleura to Dura' is often quoted as being the anatomical area that is within the remit of the Otolaryngologists. An otolaryngologist provides comprehensive medical and surgical care for patients of all ages with diseases and disorders that affect the ears, nose, throat, the respiratory and upper alimentary systems, and related structures of the head and neck. The specialty encompasses cosmetic facial reconstruction, surgery of the benign and malignant tumors of the head and neck, and the diagnosis and management of allergic, sinus, laryngeal, thyroid, and esophageal disorders.
It is important for the universities/academy to decide what knowledge and skills are critical to the basic practice of general otolaryngology, so that when our residents complete training, they are competent and confident that they can handle most of the otolaryngology problems with which their patients present, so that they know which procedures should be referred to the subspecialists. So, a 'general otolaryngologist' should be skilled enough to be a 'jack of all trades'.

Throughout the 'Globe', Otolaryngology trends towards more specialization and 'specialization is a powerful engine for change'. Our real task is to manage the changes that are occurring in a manner that will lead to safer and more effective patient care. Following the rising tide of subspecialization, specialists are now also trained in pediatric otolaryngology, otology and neurotology, rhinology, laryngology and voice disorders, allergy, head and neck surgery, facial plastic and reconstructive surgery, audiology and speech and language pathology, sleep surgery, and skull base surgery. Subspecialty training is extremely valuable, especially in cases that require an added degree of skill- such as acoustic neuroma surgery or micro-vascular free tissue transfer. Such training/fellowship enhances one's ability to provide care for patients whose cases would not be routinely seen. American Triological Society in 2015 could not conclude whether otolaryngology subspecialization increases research productivity or the quality or quantity of research in the field. But some 
otolaryngologists believe that subspecialization advances research and technology, concentrates experience, and exposes patients to more focused attention.

With the development of subspecialties, numerous tests ordered previously with a 'shotgun' approach that included all sorts of costs and ineffective methods to interpret them now became better 'targeted'. The subspecialists are more at the forefront. At the same time, there is also the issue that test results would not necessarily change treatment. For instance, genetic testing can help family planning, but not in doing anything with the patient in hand. A CT scan could show whether there is structural problem causing hearing loss, and knowing this may not lead to treatment change, it provides parents with more information about the child condition.

In Bangladesh, the specialty of 'otolaryngology' is more or less 'established' in all old government medical college hospitals. Newly established district hospital cum medical colleges is providing mainly ENT OPD and few inpatient services. We also have Bangabandhu Sheikh Mujib Medical University (BSMMU), Combined Military Hospitals $(\mathrm{CMH})$ and recently functioning National Institute of ENT (NIENT) to provide tertiary care service in ENT. Society for Assistance to Hearing Impaired Children (SAHIC), ENT and Head-Neck cancer foundation, private medical college hospitals and private corporate hospitals and clinics are the non-government organizations (NGO) also providing ENT care services. Almost all of these tertiary ENT care hospitals are providing solely 'general otolaryngology' services. Head-Neck division in BSMMU, Audiology-Vestibular department of SAHIC, and Audiology department of NIENT are the official subspecialties in Bangladesh at present with respective manpower positions but the later one is still struggling to become functioning. Despite no official subspecialist manpower position, department of ENT \& HNS, Shaheed Suhrawardy Medical College Hospital (ShSMCH) is equipped with image guided instruments, LASER, audiology \& apeech therapy unit, and a voice laboratory along with conventional otolaryngology set up. Department of ENT \& HNS of Dhaka Medical College Hospital and $\mathrm{CMH}$, Dhaka are also doing some subspecialized work like HeadNeck reconstruction and cochlear implant surgery. Otology, Rhinology and Head-Neck OPD clinics are in operation in BSMMU. With all limitations, some LASER assisted laryngeal and phonosurgery, image guided surgery, advanced endoscopic sinus surgery, cochlear implant surgery, Head-Neck reconstructive surgery are now being done in Bangladesh by our enthusiastic otolaryngologists. Recently, Army Medical Core (AMC) has trained some of their ENT manpower in different subspecialties from abroad. Till today, there has been no single residency/fellowship position and academic course/fellowship as well, in any subspecialty of otolaryngology in any academic institution in Bangladesh. Moreover, we are also lacking of auxillary manpower of subspecialization like audiologists, speech therapist, auditoryverbal therapist, physiotherapist, nutritionist, and trained nursing staff. Cost is also an important burden for our community to receive subspecialized ENT service. It is questionable whether the current cochlear implant program of BSMMU and $\mathrm{CMH}$, Dhaka would be in operation unless it is financially supported by the government of Bangladesh.

In Bangladesh, there are nearly five hundred otolaryngologists at present. Until recently, Society of Otolaryngologists and Head-Neck Surgeons of Bangladesh (ORLHNSB) was only one professional platform for the otolaryngologists of Bangladesh. Recently, 
there were inceptions of society of Head-Neck surgery, Phonosurgery, Sleep surgery, Otology, Rhinology, Endoscopic sinus and skull base surgery, Head-Neck oncology and thyroid surgery subspecialties. 'Workshop and live surgery demonstration' was also organized by some of these newly formed societies in their respective fields with the help of some renowned foreign and few local faculties. These initiatives were great and we came to learn and orient ourselves to some of the recent surgical techniques to serve our patients. Moreover, it paved a way and established some form of 'linkage' for our otolaryngologists to be trained in their field of interest in the institutes where our foreign guest faculties are attached to.

In contrast to the formation of multiple societies of different subspecialties, we shall find only a very few if ever a single subspecialized otolayngologists in their private practice and even in work place. Otolaryngologists should also change their mind set and refer the appropriate cases for subspecialized ENT care. Considering the present status of our infrastructure, manpower, residency position, trainer availability, university courses and curriculum, a very precious question may arise. Has otolaryngology subspecialization gone too far in Bangladesh to accommodate too many subspecialized societies? Should priority be given either to manpower and infrastructure development in subspecialties or to formation of the societies?

Finally, there is a concern for fragmentation within our specialty. We all should work together to have a unified voice about what are made and how they should be implemented. Concepts that sound good on paper or to the public such as 'era of subspecialty' often are not as simple as they seem and require that we all work together to make it functional and practical. There is an absolute need for subspecialization but the need for 'generalists' cannot be ignored. Patient will often self-refer to a specialist and, in otolaryngology; the physician they seek is a general otolaryngologist. So, the subspecialties should be kept under the otolaryngology 'umbrella'.

If we remember our mission of working for the best ear, nose, and throat care in treating patients, training residents, collaborating with colleagues, and working within our faculty rather than becoming the 'leader of the community', we should be able to find the correct balance between the general otolaryngologists and the subspecialists and minimize controversy in our own specialty.

\section{Md. Mazharul Shaheen \\ Associate Professor \\ Dept. of Otolaryngology \& Head-Neck Surgery \\ Shaheed Suhrawardy Medical College, Dhaka}

